\title{
Development of Cardiac Regenerative Medicine Using Human iPS Cell-derived Cardiomyocytes
}

\author{
Jun Fujita ${ }^{1,2}$ \\ ${ }^{1}$ Department of Cardiology, Keio University School of Medicine, Tokyo, Japan \\ ${ }^{2}$ Endowed Course for Advanced Heart Failure Treatment II, Keio University School of Medicine, Tokyo, Japan
}

(Received for publication on May 8, 2020)

(Revised for publication on July 28, 2020)

(Accepted for publication on July 29, 2020)

(Published online in advance on August 22, 2020)

\begin{abstract}
Heart failure is a life-threatening disease prevalent worldwide. Cardiac transplantation is the last resort for patients with severe heart failure, but donor shortages represent a critical issue. Cardiac regenerative therapy is beneficial, but it is currently unsuitable as a substitute for cardiac transplantation. Human induced pluripotent stem cells (hiPSCs) are excellent sources for the generation of terminally differentiated cells. The preparation of a large number of pure cardiomyocytes (CMs) is the major premise for translational studies. To control the quality of the generated CMs, an efficient differentiation method, purification strategy, and mass-scale culture must be developed. Metabolic purification and large-scale culture systems have been established, and pure hiPSC-derived CMs of clinical grade are now available for translational research. The most critical challenge in cell therapy is the engraftment of transplanted cells. To overcome the low engraftment ratio of single $\mathrm{CMs}$, aggregations of CMs are developed as cardiac spheroids. A cardiac transplantation device with domed tips and lateral holes has been developed for the transplantation of cardiac spheroids. Large animal models are necessary as the next step in the process toward clinical application. The transplant device has successfully been used to inject cardiac spheroids uniformly into myocardial layers in swine, and this approach is progressing toward clinical use. Remaining issues include immunological rejection and arrhythmia, which will require further investigation to establish safe and effective transplantation. This review summarizes the present status and future challenges of cardiac regenerative therapies. (DOI: 10.2302/ kjm.20200009-IR)
\end{abstract}

Keywords: heart failure, regenerative medicine, induced pluripotent stem cell, cardiomyocyte, translational research

\section{Background}

Heart failure (HF) is a life-threatening disease and the leading cause of hospitalization in many developed countries. Both the mortality and morbidity resulting from $\mathrm{HF}$ are very widespread, and the number of HF cases is increasing annually worldwide, a phenomenon referred to as the "HF pandemic." Severe HF is treated using device therapies (cardiac resynchronization therapy and implantable cardioverter defibrillator) in addition to optimal medical therapies. However, many patients eventually require ventricular assist devices and cardiac transplantation at stage D heart failure in the American Heart Association and American College of Cardiology's A-to-D staging system. ${ }^{1}$ Ventricular assist devices can cause severe infections and thrombosis, and donor shortages are a persistent challenge for cardiac transplantation. ${ }^{2,3}$ Therefore, cardiac regenerative therapies are currently used to treat patients with severe HF; however, no "standard" cell therapy has so far been established for HF. 


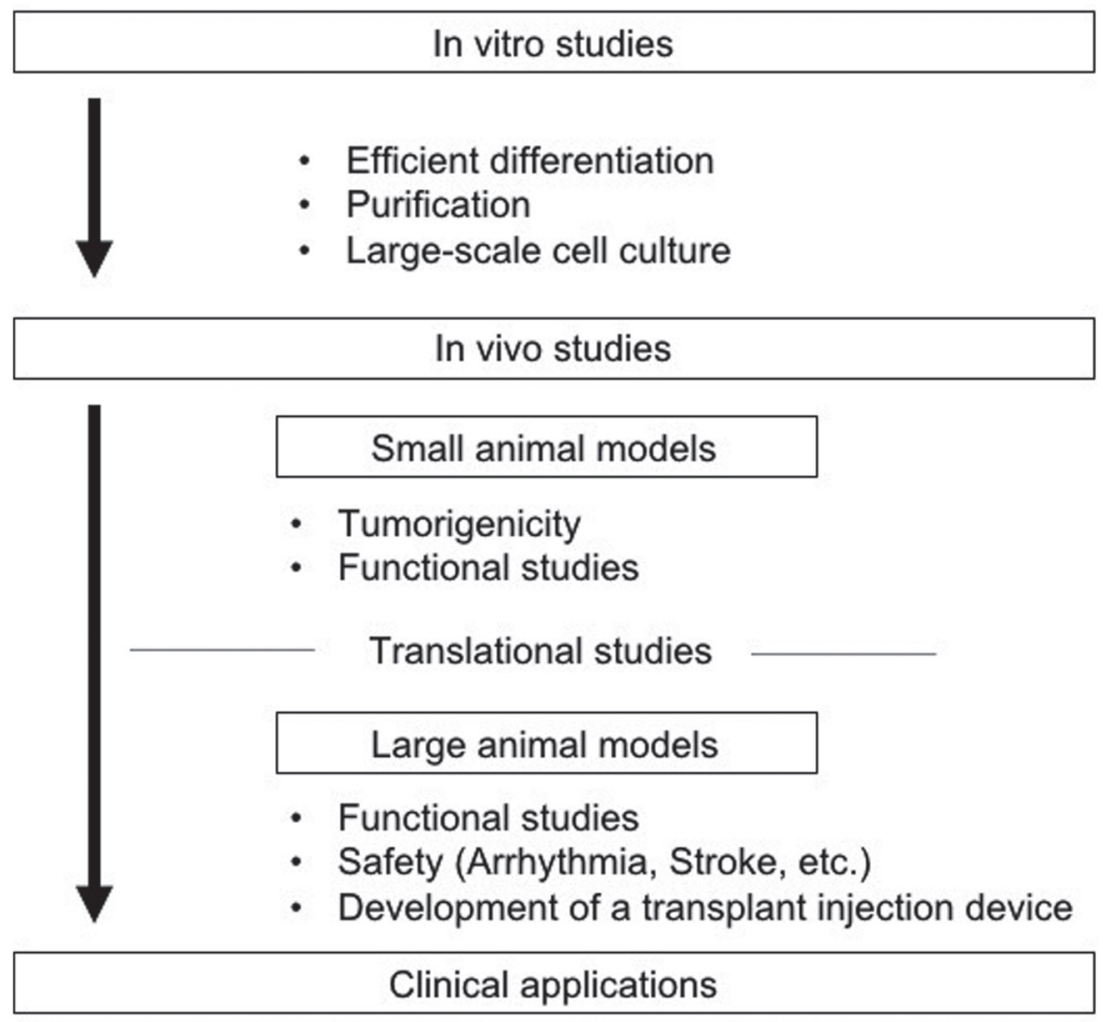

Fig. 1. Schematic of the clinical application of human-induced pluripotent stem cell (hiPSC)-derived cardiomyocytes for severe heart failure. First, clinical grade hiPSC-derived cardiomyocytes must be prepared. After assessing tumorigenicity and cardiac function, translational studies must be conducted with large animals to confirm both efficacy and safety.

Human pluripotent stem cells (human embryonic stem cells and induced pluripotent stem cells) are potent sources of human cardiomyocytes $(\mathrm{CMs})$ in vitro. ${ }^{4,5} \mathrm{Spe}-$ cifically, human induced pluripotent stem cells (hiPSCs) have strong potential to avoid immunological rejection by using patient-derived iPS cells. In recent years, the clinical application of hiPSCs has been realized in many fields (e.g., macular degenerative disease and Parkinson's disease), ${ }^{6}$ and hiPSCs also have great potential for the treatment of HF. However, a large number of hiPSC-derived CMs must be prepared in vitro for the effective improvement of cardiac function. ${ }^{7} \mathrm{CMs}$ developed in vitro are immature and it is difficult to determine whether they will function effectively along with host CMs. Therefore, transitioning from basic research to translational studies is the key to realizing clinical applications (Fig. 1).

In vivo experiments are critical for the translational approach known as "bench to bedside." Most basic research is performed on rodents (mice and rats) because they are readily available and relatively inexpensive compared with larger animals. In particular, mice are useful for studying molecular mechanisms thanks to the large number of tools available for this type of analysis. Some clinical studies have been approved after rodent experiments; however, confirmatory studies involving larger animals are desirable before clinical trials for regenerative medicine. ${ }^{8}$ The physiological characteristics of rodents are different from those of humans. For example, the heart rate is approximately 600 beats per minute (bpm) in mice and more than $300 \mathrm{bpm}$ in rats. ${ }^{9}$ The heart rate is particularly important for studies of cardiovascular diseases, because arrhythmogenicity must be assessed for clinical applications. Therefore, larger animals, such as swine, sheep, and dogs, are utilized for translational studies because their sizes are more suitable for the evaluation of medical devices, although the molecular techniques available are very limited. This review focuses on in vitro and in vivo experiments that should lead to clinical applications for cardiac regenerative medicine.

\section{hiPSC-derived CMs: differentiation, purification, and large-scale culture systems}

Cardiac differentiation from hiPSCs mirrors the developmental steps of the human heart. ${ }^{10}$ Pluripotent stem cells maintain pluripotency with core transcription factors (Oct4, Sox2, and Nanog), and their instability leads to the spontaneous differentiation of lineage cells. ${ }^{11,12}$ 
Several factors promote lineage differentiation to mesodermal cells. In particular, Wnt $/ \beta$-catenin signaling potently induces mesoderm formation. ${ }^{13}$ After mesodermal differentiation, $\mathrm{CMs}$ are sequentially developed by the inhibition of Wnt signaling. ${ }^{14}$ The differentiation ratio varies on a case-by-case basis, so that the differentiation efficiency must be evaluated for each batch. As a result, many non-CMs are also generated after differentiation, including undifferentiated and proliferating cells, leading to the formation of teratomas and other tumors. Therefore, the purification strategy is the most critical issue for exploiting hiPSC-derived CMs for clinical applications. Several purification strategies have been proposed, most of which are based on fluorescent cell sorting..$^{15}$ However, large numbers of CMs are difficult to purify using fluorescent cell sorting systems because the process is time consuming and leads to severe cell damage. We developed a purification system that utilizes metabolic differences between pluripotent stem cells and CMs. ${ }^{16,17}$ The depletion of glucose and glutamine promotes cell death in hiPSCs and non-CM derivatives, cell types that potentially cause tumor formation, whereas lactate supports the survival of CMs. After metabolic purification, less than $0.001 \%$ of hiPSCs remain. No teratomas developed when the purified CMs were subcutaneously transplanted to immunodeficient mice. Finally, massive cell culture systems are necessary for the clinical and industrial-scale production of CMs. Usually, large cell culture systems use a suspension cell culture technique, such as a bioreactor. ${ }^{18}$ However, suspension culture involves the formation of embryoid body-like masses from hiPSCs, and these tend to initiate spontaneous differentiation. Therefore, a two-dimensional (2D) culture system is a fundamental requirement for maintaining the pluripotency of hiPSCs. We developed a large-scale culture system for hiPSCs and hiPSC-derived CMs with multilayer culture plates and active gas ventilation. ${ }^{19}$ This $2 \mathrm{D}$ culture system can generate $1.5 \times 10^{9}$ hiPSC-derived CMs efficiently and sequentially. To reduce the cost of large-scale culturing, a new cell culture coating system must be developed. UV/ozone effectively modifies the surfaces of cell culture plastics and drastically reduces the amount of matrix coating. ${ }^{20}$ Adherent cells are relatively difficult to collect in multilayer cell culture plates because mechanical stimuli cannot be applied to all layers evenly, and prolonged incubation with detaching solutions causes severe cell damage. To overcome these disadvantages, we also developed an effective cell detachment system that uses resonance vibrations. ${ }^{21}$ This approach reduces the amount of detaching solution and yields a high number of hiPSC-derived CMs that are ready for use in translational studies.

\section{Transplantation strategies: cardiac spheroids and a transplant injection device}

The transplantation strategy is a key factor in cardiac cell therapy. Single CMs exhibit limited survival in host hearts. Additionally, the engraftment ratio of transplanted single CMs is very low. We found that only approximately $3 \%$ of transplanted cells survived in mouse hearts. ${ }^{22}$ Most cells were pumped out by the beating hearts. These results led to the development of improved transplantation strategies. Cell sheets are the most popular cell transplantation technique in regenerative medicine. A cell sheet is relatively noninvasive because it is simply placed on the surface of the heart. However, the application of more than three layers can result in ischemia, making it difficult to synchronize the host heart. ${ }^{23}$ To improve the engraftment ratio and cell survival, the cardiac spheroid technique was developed at Keio university. ${ }^{24}$ The efficient production of hiPSC-derived cardiac spheroids was also established by using specific microwell plates. ${ }^{25} \mathrm{Pu}-$ rified hiPSC-derived CMs were reaggregated for 7 days in the microwell plates. These spheroids had a diameter of approximately $200 \mu \mathrm{m}$ and comprised approximately 1000 pure hiPSC-derived CMs. Fluorescent beads were injected into the swine myocardial layer to determine the most effective diameter for the cardiac spheroids. The $175-\mu \mathrm{m}$ beads showed a better retention rate compared with the $20-\mu \mathrm{m}$ beads, suggesting the better retention of cardiac spheroids compared with single CMs. In summary, cardiac spheroids have the potential to be an efficient intramyocardial transplantation strategy to improve the engraftment rate of hiPSC-derived CMs.

Next, we developed a cardiac injection device specifically for the transplantation of hiPSC-derived cardiac spheroids (Fig. 2). For ideal cell engraftment, cardiac spheroids must be distributed uniformly in all myocardial layers. Transplanted cells that were injected using a single needle condensed at only one point in the myocardium, and the condensed hiPSC-CMs were exposed to a strong risk of ischemia. Consequently, the cardiac transplant device was designed to have six needles with domed tips and several lateral holes. The domed tips minimize myocardial injuries. hiPSC-derived cardiac spheroids injected from the lateral holes were distributed uniformly in all myocardial layers. Furthermore, to prevent perforation of the myocardial wall, the needles were angled at $45^{\circ}$ to the cardiac surface so that they could not be inserted deeper than $7 \mathrm{~mm}$. Finally, the transplant device was tested in swine hearts. Tissue-marking dye and cardiac spheroids were injected using the device and were found to be distributed uniformly in the myocardial layers. The retention rate of transplanted cardiac spheroids was much higher when using this device than when using single needles. These findings show that the combination of cardiac spheroids and a transplant device successfully improves the engraftment rate of hiPSC-derived CMs.

\section{Clinical studies with hiPSC-derived CMs}

The above-described successful in vitro and in vivo 

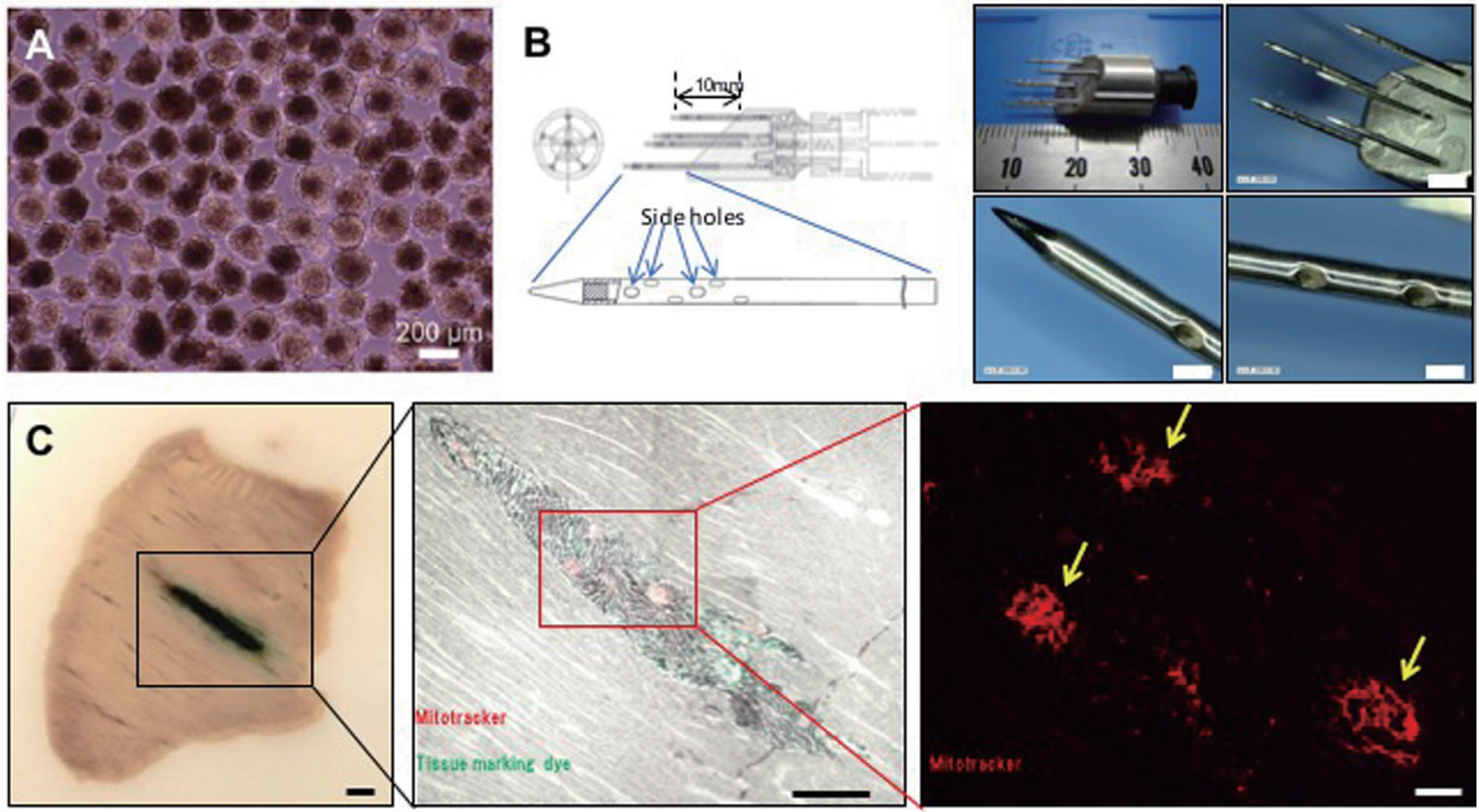

Fig. 2. Transplantation strategy with cardiac spheroids and a transplant injection device. (A) Cardiac spheroids comprise approximately 1000 pure human-induced pluripotent stem cell-derived cardiomyocytes. (B) A transplant injection device with domed tips and lateral holes. (C) Tissue-marking dye (green) and cardiac spheroids labeled with MitoTracker (arrows) were successfully distributed in swine left ventricles by the transplant injection device. Modified figure taken from Tabei et al., ${ }^{25}$ Copyright Elsevier (2020).

studies have provided a sound basis for clinical studies. Because the most critical steps have been overcome, hiPSC-derived cardiac spheroids are currently ready for clinical application (Fig. 3). The final challenge to overcome is immunological rejection. There are several ways to achieve immunological tolerance. The first and most effective strategy is the auto-transplantation of patientderived hiPSC-derived CMs. This is the theoretically ideal method, but it is practically difficult because the generation of clinical-grade iPSCs is very expensive. Clinical-grade iPSCs must have a high differentiation capacity and no genetic abnormalities. Each hiPSC derivative must be examined for tumorigenicity in vitro and in vivo. The second strategy for acquiring immunological tolerance involves the use of human leukocyte antigen (HLA)-matched iPSCs, which are especially suitable for the Japanese population. ${ }^{26}$ It was previously reported that 50 iPSC lines would be adequate to cover a three-locus match (HLA-A, B, DR) in $90.7 \%$ of the Japanese population. Some high-frequency HLA haplotype-homozygous iPSC lines are currently available for clinical application at the Center for iPS Cell Research and Application, Kyoto University. The third strategy involves the use of immunosuppressive agents. However, no data are currently available for the most effective regimen for the transplantation of hiPSC-derived CMs. From these immunological concerns, the idea of universal donor PSCs (HLA-knockout PSCs) has emerged. ${ }^{27}$ The critical challenge limiting the adoption of this outstanding strategy is how to avoid the natural killer cell-mediated missing-self responses against HLA-knockout PSCs. Retaining HLA$\mathrm{C}$ has been shown to help HLA-knockout PSC derivatives evade natural killer cell-dependent lysis. ${ }^{28}$

\section{Discussion}

Since hiPSCs were first reported, several projects have been undertaken to address their clinical application. hiPSCs have been used clinically against macular degenerative disorder. ${ }^{6}$ Because of the unique nature of the heart, however, a large number of $\mathrm{CMs}$ are required for cardiac regenerative therapies. As this review has stated, most of the challenges related to clinical application have already been resolved; however, some still remain. The maturation of $\mathrm{CMs}$ is the most critical remaining concern. Current protocols can generate ventricle-type CMs, but they have only the fetal phenotype. Their contraction force and expression of ion channels are very immature. Three-dimensional cardiac tissues that can be stimulated mechanically and electrically may be a key to acquiring 


\section{Cardiac Regenerative Medicine with human iPS cells}

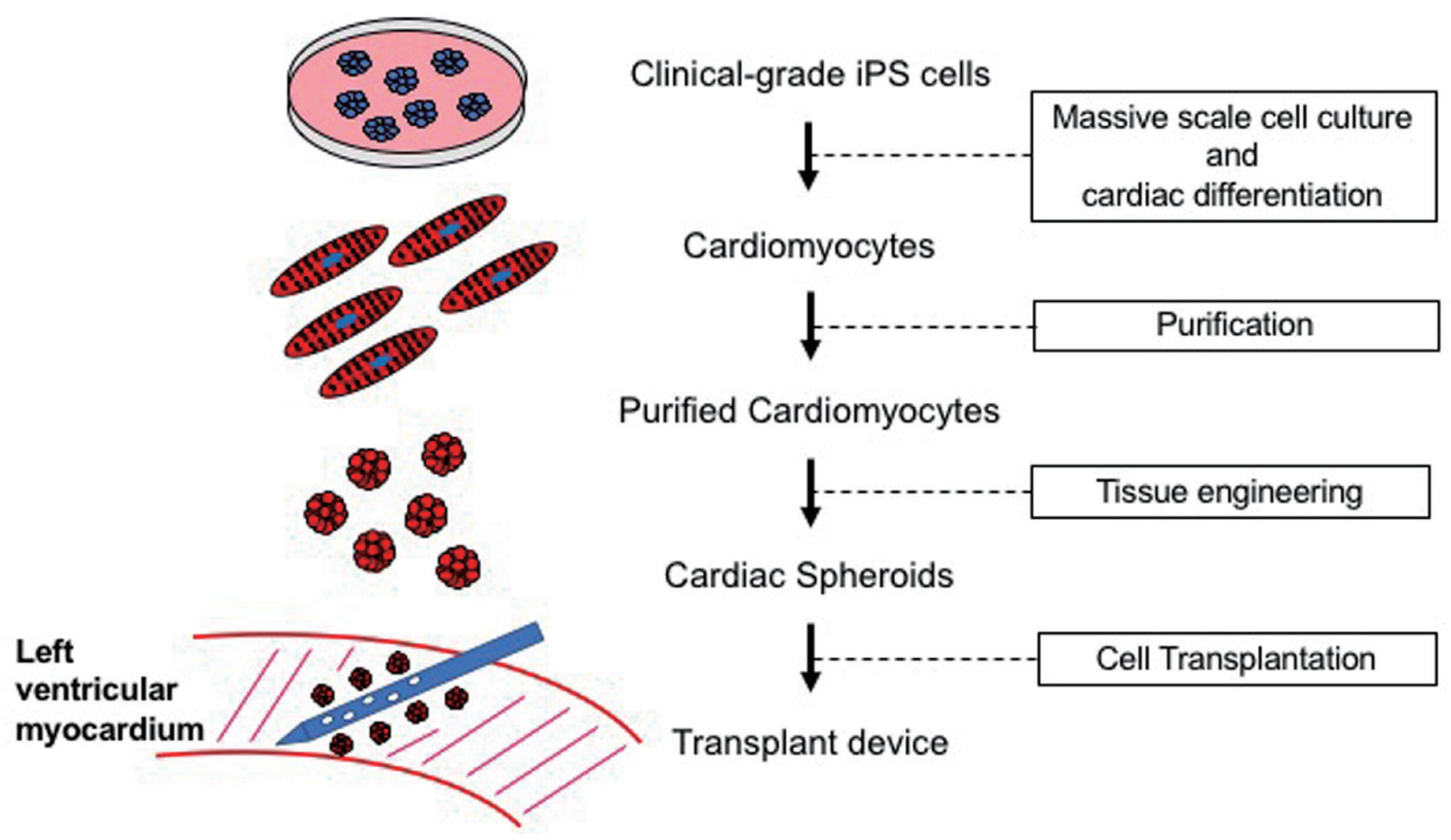

Fig. 3. Critical steps for cardiac regenerative medicine with hiPSCs. Several important tasks must be achieved step by step for the clinical application of hiPSCs.

mature cardiomyocytes. ${ }^{29}$ Organoid formation can become very advanced and may take the place of spheroids. In terms of present technology, pure CMs are the best form for clinical application. A mix of proliferative cells, such as endothelial cells and fibroblasts, could increase the risk of tumorigenesis. When the growth of vascular and interstitial cells is controlled in the future, highquality organoids with vascular formations could help improve the engraftment ratio.

Tumor formation and arrhythmia are the most significant concerns in cardiac regenerative medicine involving hiPSC-derived CMs. Metabolic purification systems can successfully purify hiPSC-derived CMs; however, tumorigenesis still raises concerns because hiPSCs are artificially generated, and genetic variance could induce the activation of cancer-driver genes. Even if CMs are purified completely, there is no guarantee that hiPSC-CMs will not cause tumor formation as a result of transformations 10 or 20 years later; however, this possibility appears to be low because fully differentiated CMs should lose their proliferative potential.

Arrhythmia is a common cardiac disorder, and transplanted hiPSC-derived CMs can cause fatal arrhythmias in patients with severe HF. Arrhythmia can occur through automaticity, triggered activity, and reentry. In addition to these fundamental mechanisms, cell transplantation causes inflammation in the host heart, which potentially aggravates arrhythmia. It has been reported that hiPSCderived CMs can synchronize with a host heart but may induce arrhythmia, including ventricular tachycardia. ${ }^{30,31}$ These results suggest that we must be prepared for arrhythmia in patients with severe HF in a clinical setting. The accumulation of preclinical data with small and large animal models is necessary for the establishment of effective and safe cardiac regenerative medicine.

\section{Conclusion}

To realize cardiac cell therapies, in addition to in vitro studies, in vivo experiments are critical as translational studies to ensure safety and efficacy. Small to large animals must be used in accordance with the experimental purposes. Conclusive results from animal studies will accelerate clinical applications and promote further progress of regenerative medicine. 
Fujita J: Transplantation of cardiac spheroids

\section{Acknowledgments}

This work was supported by Grants-in-Aid for Scientific Research from the Ministry of Education, Culture, Sports, Science and Technology of Japan (19H03660, 19K22625 and 19H01178).

\section{Conflicts of Interest}

The author owns equity in Heartseed Inc. The author is one of inventors named in patent 6333378 .

\section{References}

1. Jessup M, Brozena S: Heart failure. N Engl J Med 2003; 348: 2007-2018. PMID:12748317, DOI:10.1056/NEJMra021498

2. Khush KK, Cherikh WS, Chambers DC, Harhay MO, Hayes D, Hsich E, Meiser B, Potena L, Robinson A, Rossano JW, Sadavarte A, Singh TP, Zuckermann A, Stehlik J, International Society for Heart and Lung Transplantation: The International Thoracic Organ Transplant Registry of the International Society for Heart and Lung Transplantation: thirty-sixth adult heart transplantation report - 2019; focus theme: donor and recipient size match. J Heart Lung Transplant 2019; 38: 1056-1066. PMID:31548031, DOI:10.1016/j.healun.2019.08.004

3. Miller L, Birks E, Guglin M, Lamba H, Frazier OH: Use of ventricular assist devices and heart transplantation for advanced heart failure. Circ Res 2019; 124: 1658-1678. PMID:31120817, DOI:10.1161/CIRCRESAHA.119.313574

4. Thomson JA, Itskovitz-Eldor J, Shapiro SS, Waknitz MA, Swiergiel JJ, Marshall VS, Jones JM: Embryonic stem cell lines derived from human blastocysts. Science 1998; 282: 1145-1147. PMID:9804556, DOI:10.1126/science.282.5391.1145

5. Takahashi K, Tanabe K, Ohnuki M, Narita M, Ichisaka T, Tomoda $\mathrm{K}$, Yamanaka S: Induction of pluripotent stem cells from adult human fibroblasts by defined factors. Cell 2007; 131: 861-872. PMID:18035408, DOI:10.1016/j.cell.2007.11.019

6. Mandai M, Watanabe A, Kurimoto Y, Hirami Y, Morinaga C, Daimon T, Fujihara M, Akimaru H, Sakai N, Shibata Y, Terada M, Nomiya Y, Tanishima S, Nakamura M, Kamao H, Sugita S, Onishi A, Ito T, Fujita K, Kawamata S, Go MJ, Shinohara C, Hata K, Sawada M, Yamamoto M, Ohta S, Ohara Y, Yoshida K, Kuwahara J, Kitano Y, Amano N, Umekage M, Kitaoka F, Tanaka A, Okada C, Takasu N, Ogawa S, Yamanaka S, Takahashi M: Autologous induced stem-cell-derived retinal cells for macular degeneration. N Engl J Med 2017; 376: 1038-1046. PMID:28296613, DOI:10.1056/NEJMoa1608368

7. Murry CE, Reinecke H, Pabon LM: Regeneration gaps. J Am Coll Cardiol 2006; 47: 1777-1785. PMID:16682301, DOI:10.1016/j. jacc.2006.02.002

8. Chamuleau SA, van der Naald M, Climent AM, Kraaijeveld AO, Wever KE, Duncker DJ, Fernández-Avilés F, Bolli R, Transnational Alliance for Regenerative Therapies in Cardiovascular Syndromes (TACTICS) Group: Translational research in cardiovascular repair. Circ Res 2018; 122: 310-318. PMID:29348252, DOI:10.1161/CIRCRESAHA.117.311565

9. Levine HJ: Rest heart rate and life expectancy. J Am Coll Cardiol 1997; 30: 1104-1106. PMID:9316546

10. Fujita J, Tohyama S, Kishino Y, Okada M, Morita Y: Concise review: genetic and epigenetic regulation of cardiac differentiation from human pluripotent stem cells. Stem Cells 2019; 37: 992 1002. PMID:31021504, DOI:10.1002/stem.3027
11. Fujita J, Crane AM, Souza MK, Dejosez M, Kyba M, Flavell RA, Thomson JA, Zwaka TP: Caspase activity mediates the differentiation of embryonic stem cells. Cell Stem Cell 2008; 2: 595-601. PMID:18522852, DOI:10.1016/j.stem.2008.04.001

12. Li M, Belmonte JC: Ground rules of the pluripotency gene regulatory network. Nat Rev Genet 2017; 18: 180-191. PMID:28045100, DOI:10.1038/nrg.2016.156

13. Lian X, Hsiao C, Wilson G, Zhu K, Hazeltine LB, Azarin SM, Raval KK, Zhang J, Kamp TJ, Palecek SP: Cozzarelli Prize Winner: robust cardiomyocyte differentiation from human pluripotent stem cells via temporal modulation of canonical Wnt signaling. Proc Natl Acad Sci USA 2012; 109: E1848-E1857. PMID:22645348, DOI:10.1073/pnas.1200250109

14. Willems E, Spiering S, Davidovics H, Lanier M, Xia Z, Dawson M, Cashman J, Mercola M: Small-molecule inhibitors of the Wnt pathway potently promote cardiomyocytes from human embryonic stem cell-derived mesoderm. Circ Res 2011; 109: 360-364. PMID:21737789, DOI:10.1161/CIRCRESAHA.111.249540

15. Dubois NC, Craft AM, Sharma P, Elliott DA, Stanley EG, Elefanty AG, Gramolini A, Keller G: SIRPA is a specific cellsurface marker for isolating cardiomyocytes derived from human pluripotent stem cells. Nat Biotechnol 2011; 29: 1011-1018. PMID:22020386, DOI:10.1038/nbt.2005

16. Tohyama S, Hattori F, Sano M, Hishiki T, Nagahata Y, Matsuura T, Hashimoto H, Suzuki T, Yamashita H, Satoh Y, Egashira T, Seki T, Muraoka N, Yamakawa H, Ohgino Y, Tanaka T, Yoichi M, Yuasa S, Murata M, Suematsu M, Fukuda K: Distinct metabolic flow enables large-scale purification of mouse and human pluripotent stem cell-derived cardiomyocytes. Cell Stem Cell 2013; 12: 127-137. PMID:23168164, DOI:10.1016/j.stem.2012.09.013

17. Tohyama S, Fujita J, Hishiki T, Matsuura T, Hattori F, Ohno R, Kanazawa H, Seki T, Nakajima K, Kishino Y, Okada M, Hirano A, Kuroda T, Yasuda S, Sato Y, Yuasa S, Sano M, Suematsu M, Fukuda K: Glutamine oxidation is indispensable for survival of human pluripotent stem cells. Cell Metab 2016; 23: 663-674. PMID:27050306, DOI:10.1016/j.cmet.2016.03.001

18. Kempf H, Andree B, Zweigerdt R: Large-scale production of human pluripotent stem cell derived cardiomyocytes. Adv Drug Deliv Rev 2016; 96: 18-30. PMID:26658242, DOI:10.1016/j. addr.2015.11.016

19. Tohyama S, Fujita J, Fujita C, Yamaguchi M, Kanaami S, Ohno R, Sakamoto K, Kodama M, Kurokawa J, Kanazawa H, Seki T, Kishino Y, Okada M, Nakajima K, Tanosaki S, Someya S, Hirano A, Kawaguchi S, Kobayashi E, Fukuda K: Efficient large-scale 2D culture system for human induced pluripotent stem cells and differentiated cardiomyocytes. Stem Cell Reports 2017; 9: 14061414. PMID:28988990, DOI:10.1016/j.stemcr.2017.08.025

20. Kasai K, Tohyama S, Suzuki H, Tanosaki S, Fukuda K, Fujita J, Miyata S: Cost-effective culture of human induced pluripotent stem cells using UV/ozone-modified culture plastics with reduction of cell-adhesive matrix coating. Mater Sci Eng C 2020; 111: 110788. PMID:32279811, DOI:10.1016/j.msec.2020.110788

21. Terao Y, Kurashina Y, Tohyama S, Fukuma Y, Fukuda K, Fujita J, Takemura K: An effective detachment system for human induced pluripotent stem cells cultured on multilayered cultivation substrates using resonance vibrations. Sci Rep 2019; 9: 15655. PMID:31666563, DOI:10.1038/s41598-019-51944-w

22. Hattan N, Kawaguchi H, Ando K, Kuwabara E, Fujita J, Murata M, Suematsu M, Mori H, Fukuda K: Purified cardiomyocytes from bone marrow mesenchymal stem cells produce stable intracardiac grafts in mice. Cardiovasc Res 2005; 65: 334-344. PMID:15639472, DOI:10.1016/j.cardiores.2004.10.004

23. Fujita J, Itabashi Y, Seki T, Tohyama S, Tamura Y, Sano M, Fukuda K: Myocardial cell sheet therapy and cardiac function. Am J Physiol Heart Circ Physiol 2012; 303: H1169-H1182. PMID:23001836, DOI:10.1152/ajpheart.00376.2012 
24. Hattori F, Chen H, Yamashita H, Tohyama S, Satoh Y, Yuasa S, Li W, Yamakawa H, Tanaka T, Onitsuka T, Shimoji K, Ohno Y, Egashira T, Kaneda R, Murata M, Hidaka K, Morisaki T, Sasaki E, Suzuki T, Sano M, Makino S, Oikawa S, Fukuda K: Nongenetic method for purifying stem cell-derived cardiomyocytes. Nat Methods 2010; 7: 61-66. PMID:19946277, DOI:10.1038/ nmeth. 1403

25. Tabei R, Kawaguchi S, Kanazawa H, Tohyama S, Hirano A, Handa N, Hishikawa S, Teratani T, Kunita S, Fukuda J, Mugishima Y, Suzuki T, Nakajima K, Seki T, Kishino Y, Okada M, Yamazaki M, Okamoto K, Shimizu H, Kobayashi E, Tabata Y, Fujita J, Fukuda K: Development of a transplant injection device for optimal distribution and retention of human induced pluripotent stem cell-derived cardiomyocytes. J Heart Lung Transplant 2019; 38: 203-214. PMID:30691596, DOI:10.1016/j.healun.2018.11.002

26. Nakatsuji N, Nakajima F, Tokunaga K: HLA-haplotype banking and iPS cells. Nat Biotechnol 2008; 26: 739-740. PMID:18612291, DOI:10.1038/nbt0708-739

27. Gornalusse GG, Hirata RK, Funk SE, Riolobos L, Lopes VS, Manske G, Prunkard D, Colunga AG, Hanafi LA, Clegg DO, Turtle C, Russell DW: HLA-E-expressing pluripotent stem cells escape allogeneic responses and lysis by NK cells. Nat Biotechnol 2017; 35: 765-772. PMID:28504668, DOI:10.1038/nbt.3860
28. Xu H, Wang B, Ono M, Kagita A, Fujii K, Sasakawa N, et al. Targeted disruption of HLA genes via CRISPR-Cas9 generates iPSCs with enhanced immune compatibility. Cell Stem Cell 2019; $24: 566-578$ e7.

29. Zimmermann WH, Schneiderbanger K, Schubert P, Didié M, Münzel F, Heubach JF, Kostin S, Neuhuber WL, Eschenhagen T: Tissue engineering of a differentiated cardiac muscle construct. Circ Res 2002; 90: 223-230. PMID:11834716, DOI:10.1161/ hh0202.103644

30. Chong JJ, Yang X, Don CW, Minami E, Liu YW, Weyers JJ, Mahoney WM, Van Biber B, Cook SM, Palpant NJ, Gantz JA, Fugate JA, Muskheli V, Gough GM, Vogel KW, Astley CA, Hotchkiss CE, Baldessari A, Pabon L, Reinecke H, Gill EA, Nelson V, Kiem HP, Laflamme MA, Murry CE: Human embryonic-stem-cellderived cardiomyocytes regenerate non-human primate hearts. Nature 2014; 510: 273-277. PMID:24776797, DOI:10.1038/nature13233

31. Shiba Y, Fernandes S, Zhu WZ, Filice D, Muskheli V, Kim J, Palpant NJ, Gantz J, Moyes KW, Reinecke H, Van Biber B, Dardas T, Mignone JL, Izawa A, Hanna R, Viswanathan M, Gold JD, Kotlikoff MI, Sarvazyan N, Kay MW, Murry CE, Laflamme MA: Human ES-cell-derived cardiomyocytes electrically couple and suppress arrhythmias in injured hearts. Nature 2012; 489: $322-$ 325. PMID:22864415, DOI:10.1038/nature11317 